\title{
1 Synchrony of Non-isochronous Signals in an Acoustically Communicating Katydid
}

\section{Vivek Nityananda ${ }^{1^{*}}$ and Rohini Balakrishnan ${ }^{2}$}

3 1. Biosciences Institute, Faculty of Medical Sciences, Henry Wellcome Building, Newcastle University,

$4 \quad$ United Kingdom NE2 3AE

Ph: +441912086246

email: vivek.nityananda@newcastle.ac.uk

\section{Summary}

The ability to entrain to auditory stimuli has been a powerful method to investigate the comparative rhythm abilities of different animals. While synchrony to regular (isochronous) rhythms is well documented, synchrony to non-isochronous stimuli, with multiple components at unequal time intervals, is rarer. Several katydid species with isochronous calls have been shown to achieve synchrony as part of their natural calling interactions in multi-individual choruses. Yet no study so far has demonstrated synchrony in any insect with a non-isochronous call. Using natural calling behaviour and playback experiments, we investigated acoustic synchrony and the mechanisms underlying it in the katydid species Mecopoda 'Two Part Caller'.

17 This species has a complex non-isochronous call consisting of a long trill followed by two or more chirps.

18 We found that individual males synchronized trills and, to a lesser extent, chirps. Further investigation of trill synchrony showed that timing of trills is modified by external trills but not chirps. Chirp synchrony is modified by external chirps but also by trills. We suggest a two-oscillator model underlying synchrony in

21 this species and discuss the implications for the evolution of acoustic synchrony. 


\section{Introduction}

24 Several animals have a striking ability to synchronize motor activity in response to external stimuli. The study of the comparative abilities of animals to do so has been an important field of research (Cook et al., 2013; Hasegawa et al., 2011; Jao Keehn et al., 2019; Patel et al., 2009; Schachner et al., 2009; Wilson and

27 Cook, 2016). Experiments investigating these abilities often involve playback of artificial rhythmic stimuli to animals and studying their response as evidence of their rhythmic abilities. Rhythmic responses have, however also been demonstrated in studies of natural behaviour in other animals, including fireflies, frogs and katydids (bushcrickets) (Greenfield, 2002). Males of several katydid species produce calls to attract mates over a long distance (Alexander, 1967). In many of these species, males call together in group assemblages called choruses (Greenfield, 1994) and interact with each other acoustically to produce striking temporal patterns such as synchrony and alternation (Greenfield and Roizen, 1993a; Greenfield and Schul, 2008; Greenfield and Snedden, 2003; Hartbauer et al., 2005; Nityananda and Balakrishnan, 2007). Their natural behaviour thus serves as an ideal method by which to investigate their rhythmic abilities. Studies of acoustic synchrony in katydids have therefore typically focussed on the responses of individuals to natural

37 stimuli both in the context of playback and in multi-signaller choruses.

Acoustic synchrony in katydids is often imperfect and the calls of individual males lead or follow calls of other males by a few milliseconds (Greenfield and Roizen, 1993b; Hartbauer et al., 2005; Nityananda and Balakrishnan, 2007). The mechanisms underlying this synchrony differ in different species and various models have been suggested to describe the mechanisms underlying both acoustic synchrony in katydids and the closely related phenomenon of synchronous flashing in fireflies (Buck et al., 1981a; Buck et al., 1981b; Greenfield, 1994). In species of the katydid genus Mecopoda, these mechanisms have typically been

44 investigated using Phase Response Curves (PRCs), which represent the response of an internal oscillator 45 when disturbed by single chirps at different phases of the calling cycle (Hartbauer et al., 2005; Nityananda and Balakrishnan, 2007; Sismondo, 1990). A typical PRC thus demonstrates how the animal increases or decreases the length of its calling period in response to external chirps. In the species Mecopoda elongata, 
48 the slope of the PRC seems to determine whether synchrony or alternation occurs (Sismondo, 1990).

49 Simulations of duets based on PRCs also show that the male with the faster intrinsic chirp rate produces

50 chirps that predominantly lead those of a male with a slower rate (Hartbauer et al., 2005). In the species

51 Mecopoda 'Chirper', a similar approach showed that synchrony is enabled in this species through a

52 combination of chirp-by-chirp resetting and a change in intrinsic rate (Nityananda and Balakrishnan, 2007).

53 Different mechanisms thus enable synchrony in different species. All species that have been studied,

54 however, have had simple calls consisting of a single chirp repeated at a regular rhythm, that is, isochronous

55 calls. Studies investigating rhythm perception in other animals have also focussed on their ability to

56 perceive isochronous rhythms (Celma-Miralles and Toro, 2018). Evidence for entrainment to more

57 complex rhythms has been shown for vocal learners like parrots (Patel et al., 2009; Schachner et al., 2009),

58 but also more recently from sea lions (Cook et al., 2013). In katydids, synchrony has thus far not been

59 reported from species that have more complex calls and the mechanisms that might underlie such synchrony

60 remain unknown.

61 We here demonstrate acoustic synchrony between individuals of Mecopoda 'Two Part Caller', a katydid

62 species with a complex non-isochronous call consisting of a trill, followed by two or more short chirps (Fig.

63 1). This is one of five southern Indian species belonging to the genus Mecopoda that look identical but have

64 dramatically different songs (Nityananda and Balakrishnan, 2006). This song type is found in the evergreen

65 forests of the state of Karnataka in South India and has a peak breeding season between the months of

66 March and June. The call of this species consists of a verse, comprising a trill followed by a set of chirps

67 (Fig. 1). Using acoustic playback experiments, we investigated acoustic synchrony and the possible

68 mechanisms underlying it in this species. 
a)

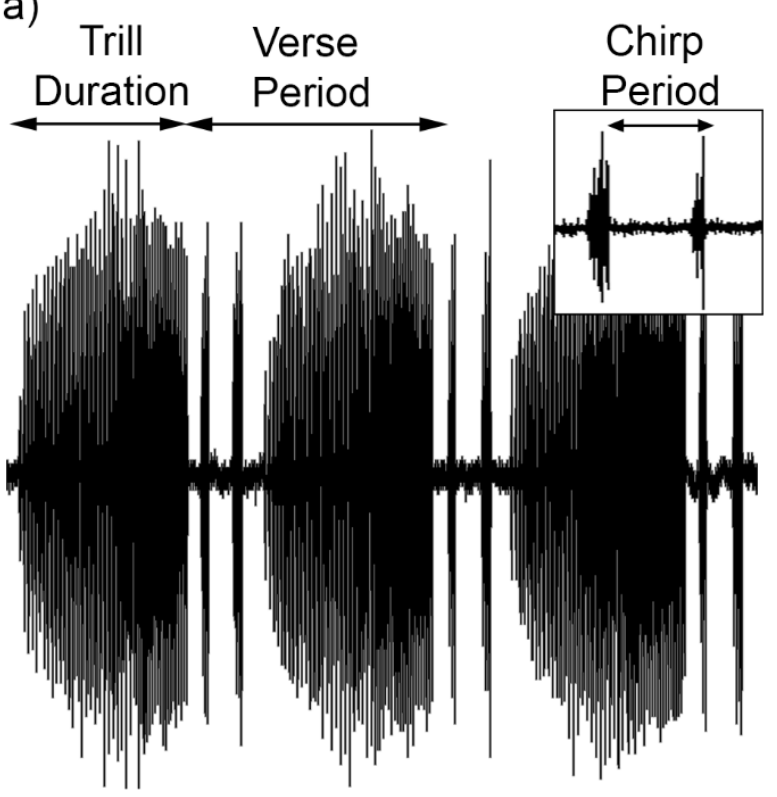

$1 \mathrm{~s}$

b)
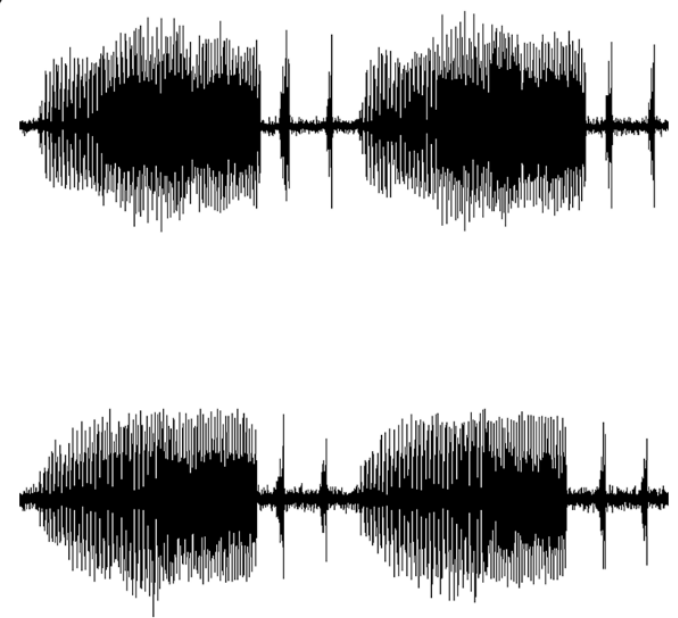

$1 \mathrm{~s}$

Fig. 1. a) Oscillogram of the call of Mecopoda 'Two Part Caller' b) Oscillogram of two simultaneously

71 calling males with synchronized trills. Inset shows a magnified view of the chirps, 
74 In all experiments, individual males were placed in acoustically transparent nylon mesh cages and the

75 calling of the males and any stimuli played back were recorded on separate channels in an anechoic chamber

$76(2 \times 2 \times 3 \mathrm{~m})$ using tiepin microphones and custom-built amplifiers placed directly in front of the cages.

77 The output of the microphones was digitized at a sampling rate of $16 \mathrm{kHz}$ using an NI-DAQ AT-MIO-16E-

782 card and the software Labview 6.0 (National Instruments Corporation, USA).

79 All playback experiments used stimuli taken from a previous recording of an individual 'Two Part Caller' 80 male made using a Bruel and Kjaer Sound Level Meter (Type 2231with a 1/4" microphone (4939: flat

81 frequency response from $4 \mathrm{~Hz}$ to $70 \mathrm{kHz}$ ) and digitized at a sampling rate of $200 \mathrm{kHz}$ using a NI-DAQ AT-

82 MIO-16E-2 card and the software Labview 6. 0 (National Instruments Corporation, USA). Stimuli were 83 played out at a sampling rate of $200 \mathrm{kHz}$ using an NI-DAQ AT-MIO-16E-2 card and an Avisoft Ultrasonic

84 Scanspeak speaker (frequency range 1-120 kHz) from a distance of $2 \mathrm{~m}$. The stimuli were played out at 91

$85 \mathrm{~dB}$ SPL (peak) $30 \mathrm{~cm}$ from the speaker measured using a Bruel and Kjaer Sound Level Meter type 2231

86 with a $1 \frac{1}{4}$ in 4939 microphone (frequency response $4-70 \mathrm{kHz}$ ).

87

88

89

90

91

92

93

94

95

96

97

\section{Song recording and analysis}

All recordings were made at night, during the natural calling time of the animals. Solo recordings were made with individuals isolated in the anechoic chamber and duets were recorded with the two cages placed

$2 \mathrm{~m}$ apart from each other in the anechoic chamber. During duets and the Phase Response Curve experiments, outputs were simultaneously obtained on two separate channels from the two microphones. Custom MATLAB (Mathworks, USA) programs were subsequently used to first obtain the time of call onsets and offsets and then to calculate the period, duration and phase relationships of the call elements. A Testo 110 thermometer (Testo Ltd., UK) was used to measure the ambient temperature during recordings. The mean temperature across all recordings was $22.23{ }^{\circ} \mathrm{C}( \pm 0.30 \mathrm{~S}$. D.).

Phase relationships between trills were calculated according to the formula

$\mathrm{P}_{\mathrm{t} 12}=\mathrm{T}_{11}-\mathrm{T}_{21} / \mathrm{T}_{22}-\mathrm{T}_{21}$ 
where $T_{11}$ is the time of offset of the trill, $T_{21}$ is the time of offset of the preceding trill of the partner and $\mathrm{T}_{22}$ is the time of offset of the following trill of the partner.

100

101

102

103

104

105

106

107

108

109

110

111

112 118 below.

\section{The effect of trill playback on trills}

\section{Phase Response Curves} every 7 verse periods.

Phase relationships of the chirps were calculated with the same formula using chirp offsets instead of trill offsets. Phase values thus obtained were multiplied by $360^{\circ}$ to obtain the phase angles. The $\mathrm{x}$ and $\mathrm{y}$ components of each of these angles were determined assuming each angle was a vector of unit length. The components for each duet were then summed together to obtain the $\mathrm{x}$ and $\mathrm{y}$ components of the mean vector for each duet. The angle and length of the mean vectors were then calculated (Batschelet, 1981). A V-test (Batschelet, 1981) was performed to test if the vectors were clustered around an angle of $0^{\circ}$ which would indicate perfect synchrony. The mean trill and chirp periods during solos and duets were compared using unpaired t-tests at a significance level $\alpha=0.05$.

To obtain the Phase Response Curves (PRCs), a previously recorded stimulus was played out after the male initiated calling. The stimulus was played out at a random phase of the male's calling, approximately once

We obtained PRCs in response to playback of both trills (Trill PRCs) and chirps (Chirp PRCs). In both trill and chirp playback experiments, we looked at the effect on both trills and chirps and obtained separate PRCS for each with calculations as described below. We thus had four types of PRCs demonstrating the effect of trill playback on trills, trill playback on chirps, chirp playback on trills and chirp playback on chirps. For all four PRCs, the stimuli played out and the calls of the male were simultaneously recorded as described above. The times of offsets and onsets were obtained and phases were calculated as detailed

For this PRC, the stimulus phase was determined according to the formula 
122 where $\mathrm{S}$ is the stimulus phase, $\mathrm{t}_{\mathrm{s}}$ is the time of offset of the stimulus trill, $\mathrm{t}_{\mathrm{fm} 1}$ was the time of offset of the

123 preceding trill of the male and $\mathrm{T}$ was the undisturbed verse period of the calling male calculated as the mean

124 of three verse periods of the male before the playback (Fig. 2a). Stimulus phases close to 0 represent stimuli

125 played out soon after the end of preceding trill and phases close to 1 represent stimuli played out close to

126 the next trill.

127 The response phase was determined according to the formula

128

$\mathrm{R}=\left(\mathrm{t}_{\mathrm{fm} 2}-\mathrm{t}_{\mathrm{fm} 1}\right) / \mathrm{T}$

(Equation 2)

where $\mathrm{R}$ is the response phase.

$\mathrm{t}_{\mathrm{fm} 1}$ was the time of offset of the trill preceding the stimulus, $\mathrm{t}_{\mathrm{fm} 2}$ was the time of offset of the following trill

of the male and $\mathrm{T}$ is the undisturbed verse period of the calling male calculated as the mean of three verse

134 playback.

\section{The effect of trill playback on chirps}

For this PRC, the calculation were made as in Equations 1 and 2 except that $\mathrm{t}_{\mathrm{fm} 1}$ was the time of offset of

137 the preceding chirp of the male, $\mathrm{t}_{\mathrm{fm} 2}$ was the time of offset of the following chirp of the male and $\mathrm{T}$ was the

138 undisturbed chirp period of the calling male calculated as the mean of three chirp periods of the male before

139 the playback. Note that when the period between two chirps included a trill, this chirp period was not used 140 to calculate the undisturbed chirp period. 
a) Trill phase calculations

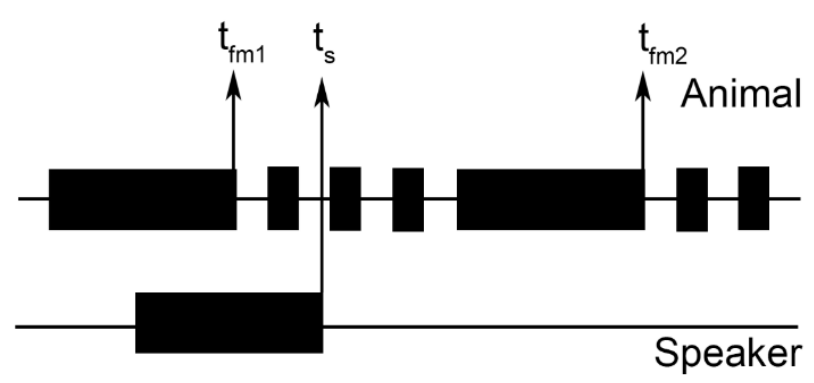

b) Chirp phase calculations

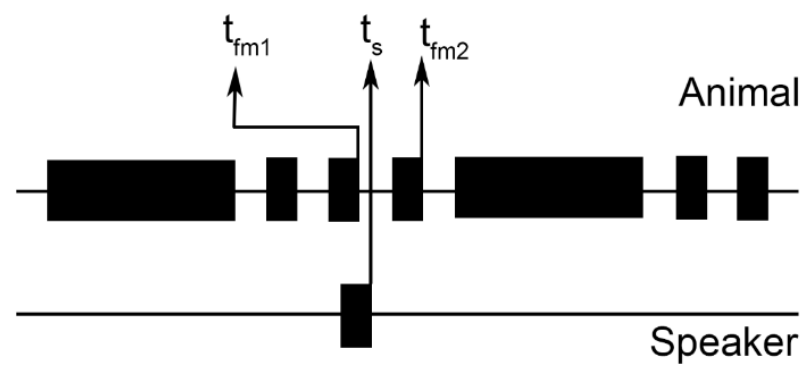

144 Fig. 2. Phase calculations for the Phase Response Curves. Long black rectangles represent trills and 145 shorter rectangles represent chirps. a) Trill phase calculations computed phase with respect to the offsets 146 of the stimulus played back from the speaker and the trills of the calling animal. Note that the speaker

147 stimulus here is depicted as a trill but in other experiments this was a chirp. b) Chirp phase calculations 148 computed phase with respect to the offsets of the stimulus played back from the speaker and the chirps of 149 the calling animal. Note that the speaker stimulus here is depicted as a chirp but in other experiments this 150 was a trill. See the main text for calculations and further details.

152 For this PRC, calculations were made using Equations 1 and 2 except that $t_{\mathrm{s}}$ was the time of offset of the 153 stimulus chirp. 
For this PRC, the calculations were made as for the effect of trill playback on chirps except that $t_{\mathrm{s}}$ was the time of offset of the stimulus chirp (Fig 2b).

157 In all four cases, the Phase Response Curve (PRC) was obtained by plotting the response phase against the 158 stimulus phase. The PRC gives us a picture of how the animal adjusts the timing of its next trill or chirp in 159 response to stimulus playback at different phases in its calling cycle.

The means of three trill periods before and after the period disturbed by stimulus playback were calculated and compared using student's t-tests with the significance level was Bonferroni corrected for multiple comparisons (Cabin and Mitchell, 2010). The tests were used to determine if there was a significant difference in trill period caused by playback of the stimulus. A similar comparison of means was made between the means of three trill periods before one stimulus playback and the next one approximately seven periods later. This enabled us to examine if the effects of stimulus playback on trill period were long-lasting. of tests in which the response of males to calls without chirps was recorded. Two categories of experiments

169 were conducted for each male: a positive control duet and a duet with chirpless calls. During the positive 170 control duets, the recording played out to the male consisted of a standard 'Two Part Caller' verse of 171 duration $1.89( \pm .11$ S.D.) s and a period of $2.05( \pm .09) \mathrm{s}$ followed by two chirps. During the chirpless

172 duets, the same call was used with the chirps removed and replaced by silence. The call onsets and offsets 173 were obtained and analyzed as previously described.

\section{Results}

176 During solo calling, individuals $(\mathrm{N}=22)$ produced verses with a mean period of $1.92 \mathrm{~s}( \pm .10 \mathrm{~S} . \mathrm{E} ., \mathrm{N}=$ $177236.77 \pm 49.79$ S.E verses per individual). These consisted of trills with a mean duration of $1.31 \mathrm{~s}( \pm .07$ 
178 S.E.) followed by chirps of mean duration of $67.8 \mathrm{~ms}( \pm 0.36$ S.E., $\mathrm{N}=272.09 \pm 82.99$ S.E chirps per

179 individual) (Figure 1a). The mean number of chirps following the trills in the solo bouts was $2.02( \pm 0.065$

180 S.E.).

181 In acoustic duets in the laboratory, individual males ( $\mathrm{N}=18$ duets, 36 males) synchronized their trills $(\mathrm{N}=$

$182144.39 \pm 26.24$ S.E. trills per focal individual), with a mean vector angle of $7.41^{\circ}$, where an angle of $0^{\circ}$ or

$183360^{\circ}$ would represent perfect synchrony (Fig. 3a,c). The vector angles across all duets were within $56^{\circ}$ of

184 perfect synchrony $\left(0^{\circ} / 360^{\circ}\right)($ Fig 3c). The length of the average mean vector across all duets was $0.92( \pm$

185.01 S.E.) and ranged from 0.76 to 0.99 indicating a high degree of synchrony (a vector length of 1 represents

186 perfect synchrony). The distribution of mean vector angles was significantly different from a uniform

187 distribution and the $\mathrm{V}$-test confirmed that the angles were centred at $0^{\circ}\left(\mathrm{V}\right.$-test, $\left.\mathrm{v}=16.39, \mathrm{P}=2.35 * 10^{-8}\right)$.

188 Males also synchronized their chirps $(\mathrm{N}=179.17 \pm 42.32$ S.E. chirps per individual); the distribution of

189 chirp phase angles was also significantly different from a uniform distribution and centred around $0^{\circ}(\mathrm{V}-$

190 test, $\mathrm{v}=11.52 \mathrm{P}=6.15^{*} 10^{-5}$, Fig. $3 \mathrm{~b}, \mathrm{~d}$ ). The degree and precision of synchrony, however, was less than

191 that of the trills (Fig. 3d) with an average mean vector length of 0.64 ( \pm .07 S.E.) and spread over a larger

192 range of angles with a maximum angle of $121.99^{\circ}$ from perfect synchrony. 

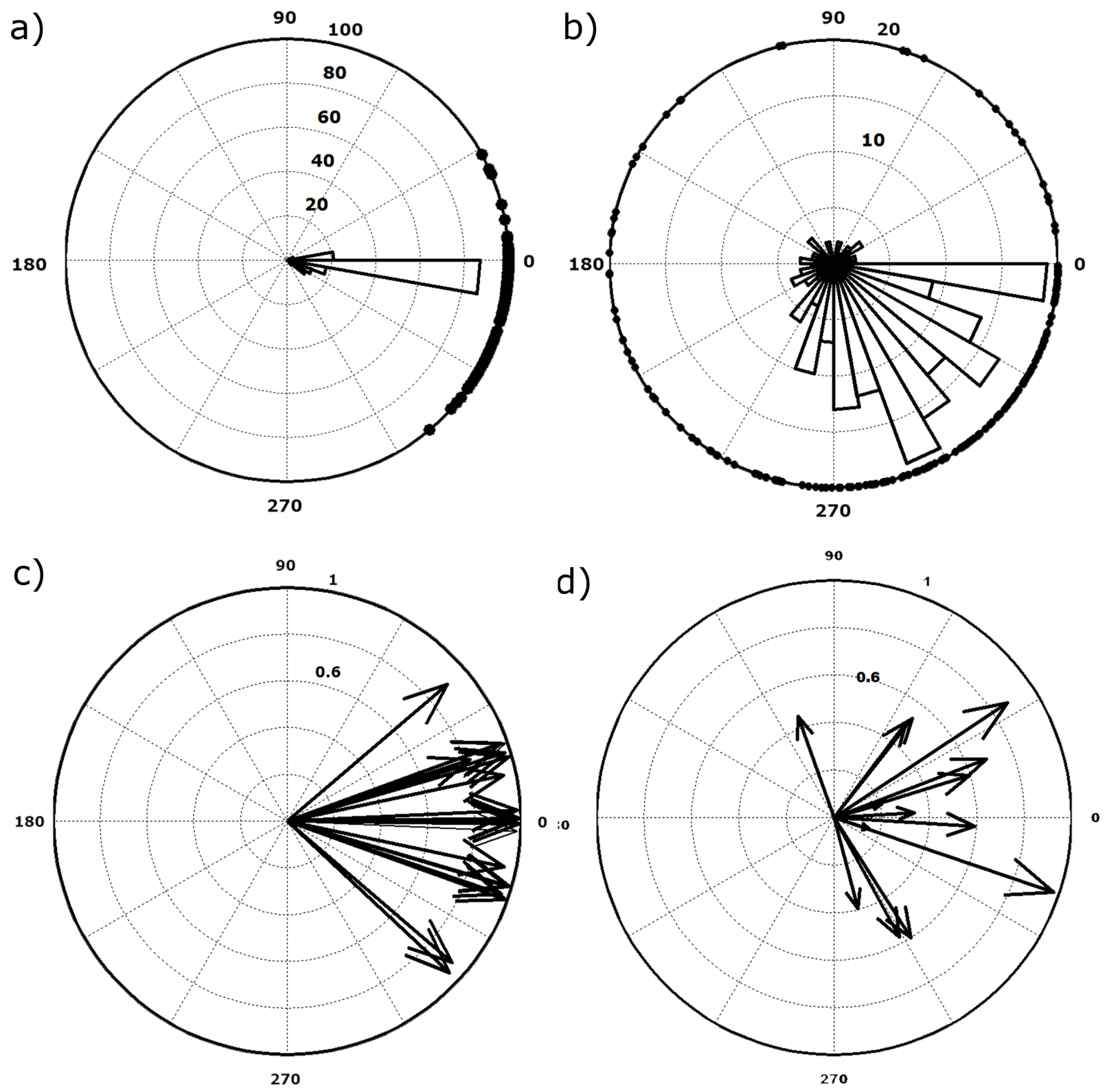

Fig. 3. Synchrony in Mecopoda 'Two Part Caller'. Top: Representative polar plots of timing relationships between a) trills and b) chirps of two calling males. Points on the circumference represent phase values. 
The verse period was significantly greater in duets than during solo calling for 9 out of 22 individuals (Fig. 4, Bonferroni corrected $t$ tests, all Ps $<0.001$, all ts $<6.02$ and $>-238.14$ ) and significantly lower for 8 individuals (Fig. 4, Bonferroni corrected t tests, all $\mathrm{Ps}<0.001$, all ts $<16.91$ and $>-4.21$ ). The mean absolute change in verse period from solos to duets was $276( \pm 60.17$ S.E. $)$ ms. The mean difference in verse period between pairs of duetting males was $48.77( \pm 19.15$ S.E.) $\mathrm{ms}$. This was much less that the mean difference between the solo verse periods of each pair (317.77 $\mathrm{ms} \pm 72.16 \mathrm{~S} . \mathrm{E})$ as might be expected because of the synchrony during duets.

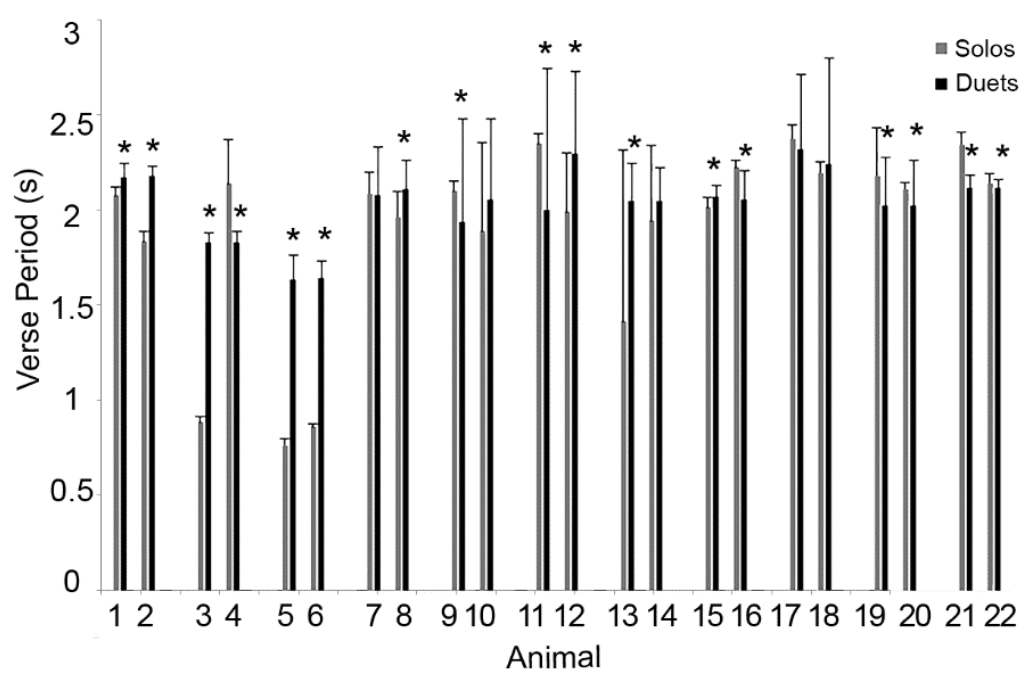

207 Fig. 4. Change in verse period from solos to pairwise interactions. Grey bars represent values obtained during solos. Black bars represent values obtained during duets. Values obtained from pairs of males that interacted with each other are grouped together and the numbers below identify individual animals. Asterisks indicate significant differences between the values obtained in solos and the values obtained in duets $(\mathrm{p}<0.0023)$.

The phase response curves of individuals rose for about half the stimulus phase after which they had a slight dip below 1 before returning to a value of 1 (Fig. 5). This indicates a delay in response to early stimulus phases and an advance in response to the later ones. Both delay and advance were however, not pronounced 
216 in all except three individuals (Fig. 5, animals 2, 3 and 9). In a few individuals, there were some trills that

217 were delayed by a large amount in response to trills in the middle stimulus phases (Fig. 5, animals 2, 4, 5

218 and 13). There was no effect of stimulus phase on the timing of subsequent trills.
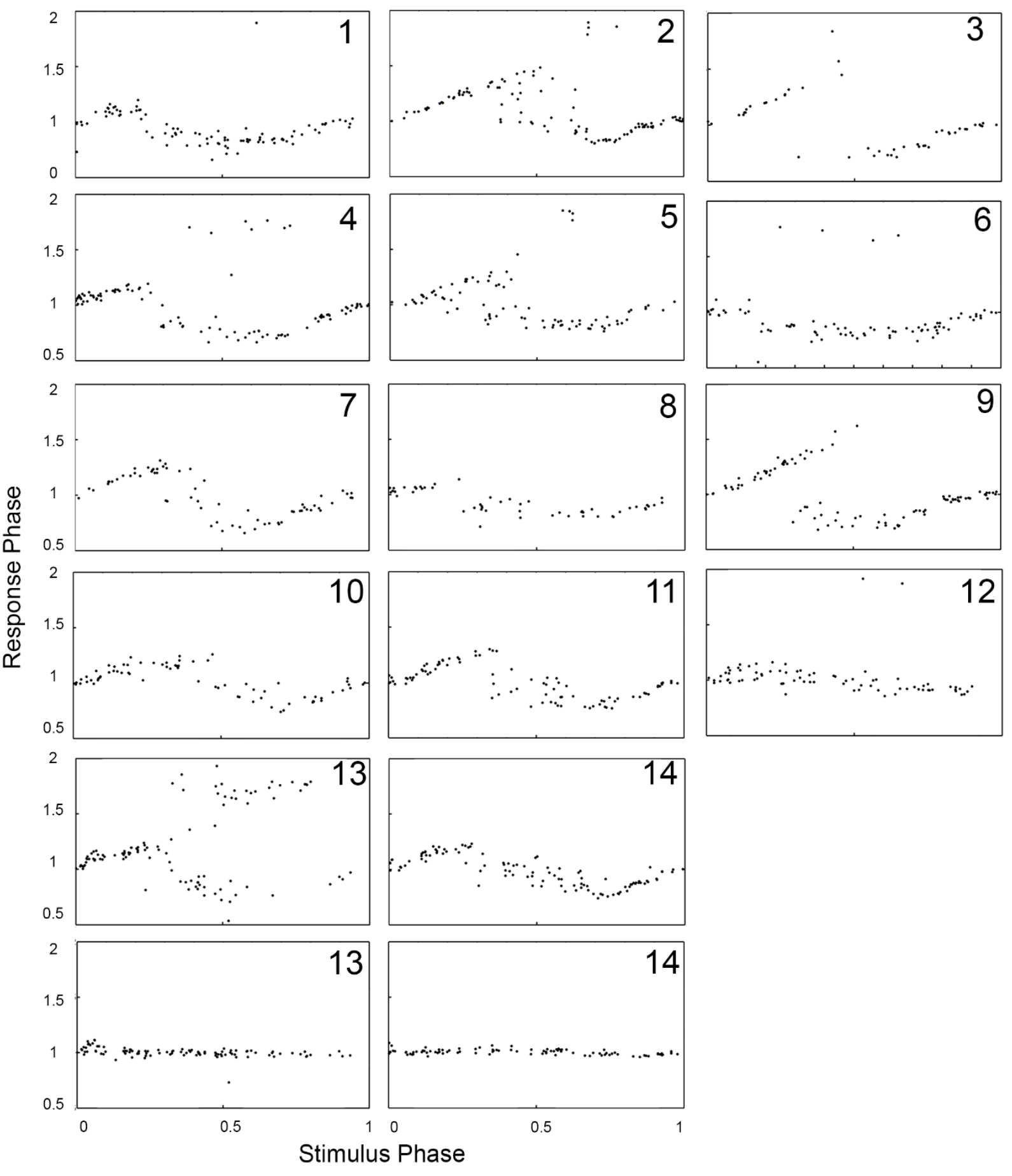
Fig. 5. Trill Phase Response Curves obtained by plotting the response phase of trills in response to playback

221 of stimulus trills. The last row depicts the phase response in the period following the disturbed period.

222 Numbers refer to individual animals.

223 An analysis of verse periods before and after the playback of stimulus trills showed that in 6 out of 14

224 individuals, the average of three periods following the trill playback was significantly lower than the 225 average of three periods preceding it (Bonferroni corrected $t$ tests, all Ps $<0.003$, all ts $>0.02$ ). The mean 226 difference between the periods before and after the stimulus trills was 64 ( $\pm 16 \mathrm{SE}) \mathrm{ms}$. There was, however, 227 no significant difference between the averages of periods preceding a stimulus trill and periods preceding 228 the subsequent stimulus trill ( $\mathrm{t}$ tests, all Ps $>0.68$, all ts $>-0.13$ and $<0.42$ ) with the mean differences 229 between these two sets being $30( \pm 17 \mathrm{SE}) \mathrm{ms}$.

231 Since it was less likely that the onset of our playback trill would fall in the chirp section of the calling verse 232 of the animal, we could not obtain individual Phase Response Curves for each animal. Instead we combined data across all animals to generate a single PRC. This PRC indicates that the playback of trills increases the period of the chirps if ending in the first half of the calling period (stimulus phase less than 0.5) (Fig. 6a).

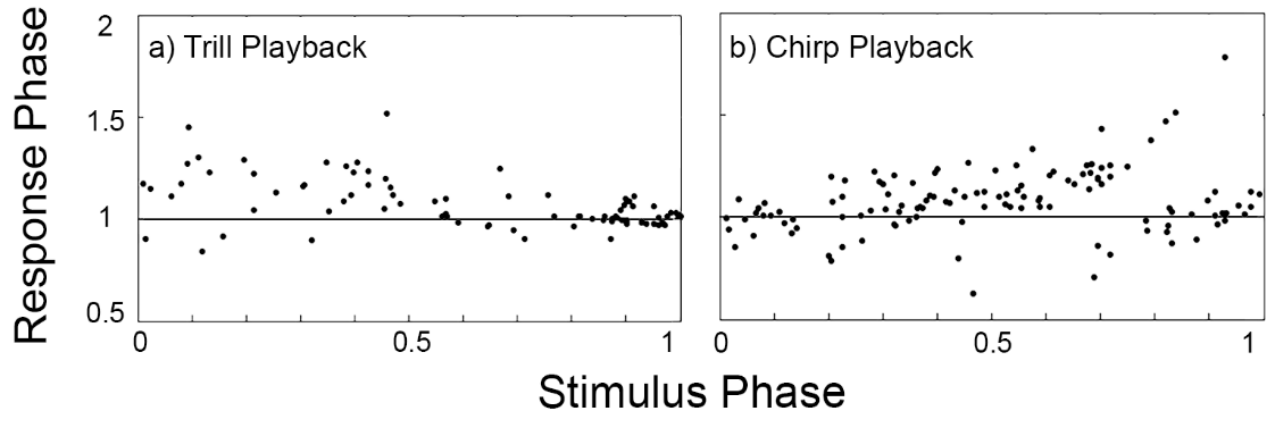


238 Fig 6. Chirp Phase Response Curves obtained by plotting the response phase of chirps in response to

239 playback of a) stimulus trills and b) stimulus chirps. Both plots combine data from multiple animals.

240 One interesting aspect of trill playback, was that the number of chirps produced by the animal was

241 significantly greater when the trill playback overlapped with the offset of the animal's trill (paired $\mathrm{t}$ test, $\mathrm{t}$

$242=4.83, \mathrm{P}=3.27 * 10^{-4}, \mathrm{~N}=14$ males $)$. The mean number of chirps produced was $2.74( \pm 0.5$ S.D.) when

243 the playback overlapped the animal's trill offset ( $\mathrm{N}=14$ males, $55.07 \pm 15.37$ playback trills per individual)

244 and $2.10( \pm 0.44$ S.D.) when the playback did not overlap $(\mathrm{N}=14$ males, $30.36 \pm 8.35$ playback trills per

245 individual).

247 Chirps played to individual males at random phases during their calls had no effect on the timing of the

248 trills of the males (Fig. 7). The PRCs obtained were flat and the response phase stayed at a value of 249 approximately 1 for all stimulus phases. The average of three verse periods before and after chirp stimuli 250 did not significantly differ for 11 out of 12 individuals ( $t$ tests, all Ps $>0.12$, all ts $>-0.27$ and $<2.16$ ) and 251 the mean difference between periods before and after the stimuli was $32( \pm 10 \mathrm{SE}) \mathrm{ms}$. 
bioRxiv preprint doi: https://doi.org/10.1101/2020.12.10.419101; this version posted April 7, 2021. The copyright holder for this preprint (which was not certified by peer review) is the author/funder, who has granted bioRxiv a license to display the preprint in perpetuity. It is made available under aCC-BY-ND 4.0 International license.

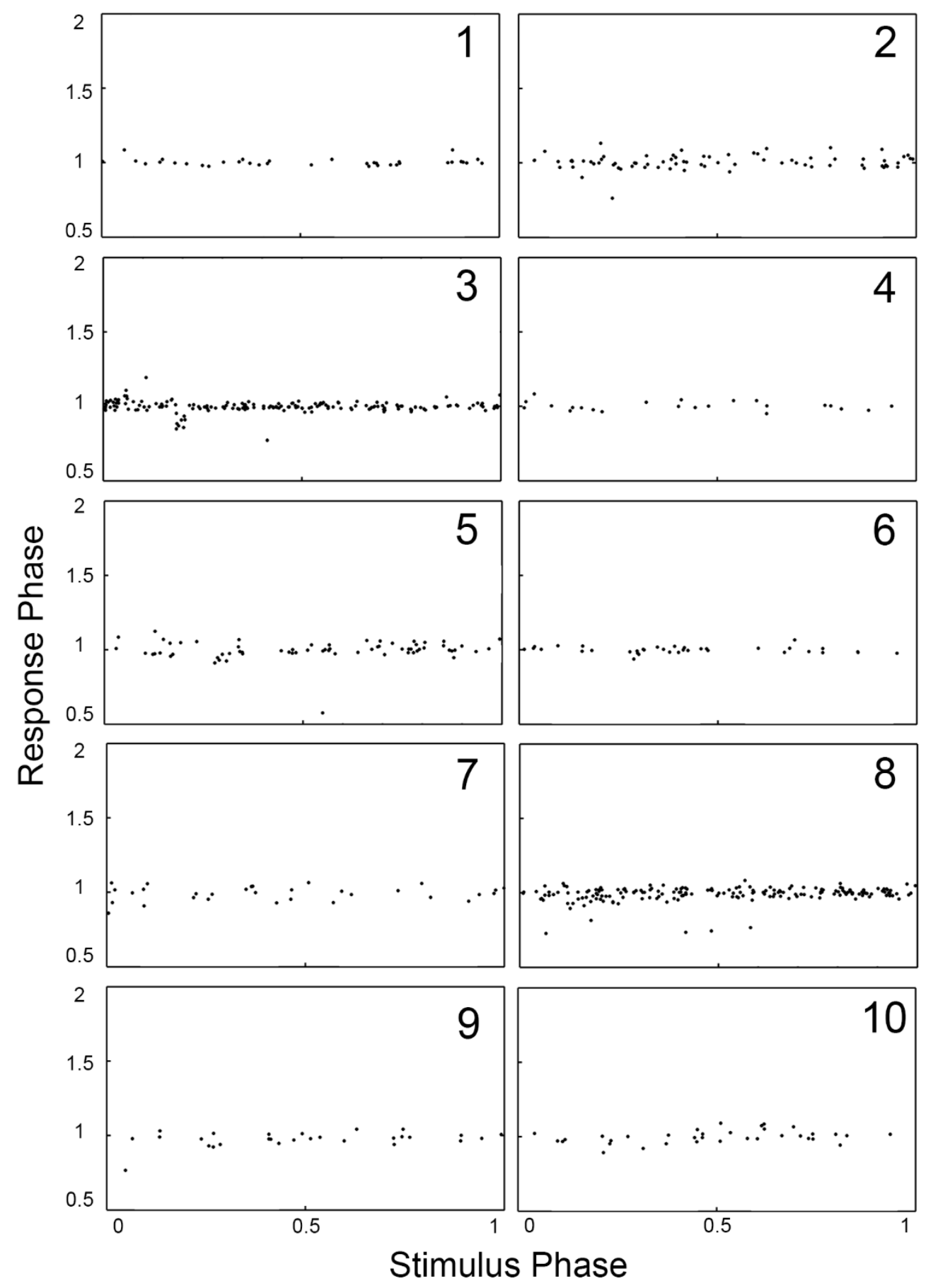


254 Fig 7. Trill Phase Response Curves obtained by plotting the response phase of trills in response to

255 playback of stimulus chirps. The last row depicts the phase response in the period following the disturbed

256 period. Numbers refer to individual animals.

257 The effect of chirp playback on chirp Phase Response Curves

258 As discussed above, due to the lack of cases when the playback chirps overlapped the animal's chirps, we

259 were only able to obtain a combined PRC across all animals. This PRC described how the animals adjusted

260 their chirp periods in response to playback of chirps (Fig. 6b). The response phase increased above 1 with

261 increasing stimulus phase up to around a stimulus phase of 0.7. After a stimulus phase of around 0.7, the

262 response phase was mostly below 1 and reach 1 at a stimulus phase of 1 . Thus, the chirp periods were

263 increased in response to chirps played back early in the chirp period, but were shortened in response to

264 chirps played back later in the chirp period.

Response to chirpless calls

26611 out of 14 males synchronised their trills with those of the positive control stimulus played out to them

267 (Fig. 8). The phase angles of the mean vectors obtained in these duets were different from a uniform

268 distribution and were centred around $0^{\circ}\left(\mathrm{V}\right.$ test, $\left.\mathrm{v}=9.36, \mathrm{P}=2.0044 * 10^{-4}\right)$. Three out of the 14 individuals,

269 however, failed to synchronize their trills and instead had a phase angle closer to $90^{\circ}$.

27012 out of 14 males also synchronized their trills with those of the stimulus (chirpless calls). The phase

271 angles of the mean vectors obtained in these duets were not uniformly distributed and were centred around

$2720^{\circ}\left(\mathrm{V}\right.$ test, $\left.\mathrm{v}=10.42, \mathrm{P}=4.11^{*} 10^{-5}\right)$. Similar to the positive control duets, two out of 14 individuals failed

273 to synchronize and had mean vector angles above $90^{\circ}$. 

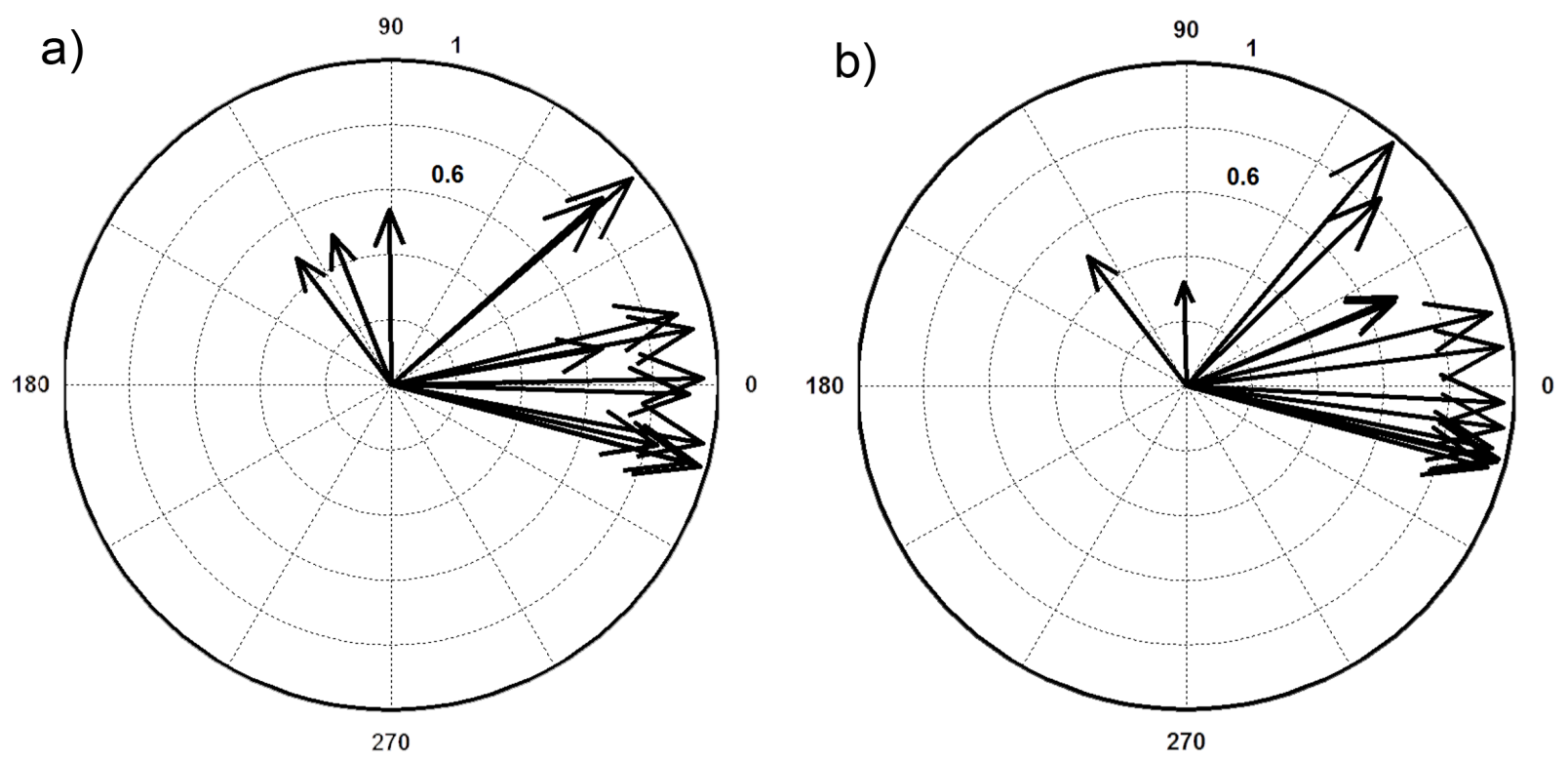

275

276

277

278

279

280

281

Fig 8. Mean phase vectors of trills during interactions with a) a positive control playback stimulus and b) a chirpless playback stimulus.

\section{Discussion}

Our results show that individuals of the species Mecopoda 'Two Part', a katydid with a complex nonisochronous call, synchronize elements of their calls. The synchrony is governed by mechanisms similar to those in other synchronizing katydid species and gives us an insight into the oscillators underlying song production in this species.

\section{Phase Response Curve comparisons}

Like several synchronizing katydid species, the Trill Phase Response Curve obtained for Mecopoda 'Two Part Caller' has both an 'advance' phase and a 'delay phase'. Similar to Mecopoda elongata, both phases affect the immediate chirp and not the following chirp. This distinguishes the PRC from that of many other well studied species like the katydids Neoconocephalus spiza and the firefly Pteroptyx cribellata (Buck et al., 1981a; Greenfield and Roizen, 1993a). In these species, the immediate chirp is only delayed, and the advance phase only affects the subsequent chirp. Previous models such as the phase advance and phase 
delay models that have been used to explain the mechanisms of synchrony are thus insufficient to explain individuals in Mecopoda 'Two Part Caller' is nonlinear and less pronounced. This PRC is perhaps more similar to that modelled for visual synchrony in other firefly species such as Pteroptyx malaccae (Ermentrout, 1991). Using one classification of firefly 'pacemakers' (Hanson, 1978), Mecopoda 'Two Part Caller' has a variable intrinsic chirp period with a small amplitude PRC. In this respect it differs from both previously studied species of the genus. Both Mecopoda elongata and Mecopoda 'Chirper' have high amplitude PRCs with two distinct arms and a break point around 70\% into the period (Hartbauer et al., 2005; Nityananda and Balakrishnan, 2007). In fact, the chirp PRC (Fig 6b) we obtained for 'Two Part Caller' fits with this pattern and resembles the PRC previously obtained for Mecopoda elongata. In contrast, the trill PRC for most individuals consists of a continuous curve without any break points. Thus, the mechanism for adjusting chirp periods resembles that of other chirping species but the mechanism for adjusting trill periods is different.

Like 'Chirper', however, 'Two Part Caller' also has a variable intrinsic chirp period. In both species, the chirp period before external acoustic stimulation differs from that after, but the difference is lost if periodic stimulation is missing. This resembles a model for firefly synchrony (Ermentrout, 1991), which assumes an adaptable free-running period that enables synchrony. Oscillators that fit this model have a period that adapts to the period of the external stimulus until the two are in synchrony. This means that whether the period of the male in duets increases or decreases will also ultimately depend on the rate changes that occur in the duetting partner, as can be seen in our results from actual duets (Fig 4). If the period of the external stimulus or duetting partner is out of the range of adaptability, the model predicts phase-locking with a small phase difference instead of synchrony. This might be more like the results

311 obtained in the positive control playback experiments of our study where all males lagged the stimulus by 312 a specific amount and some had poor synchrony. 
314 In contrast to other species from which synchrony has been reported, Mecopoda 'Two Part Caller' has a

315 call with more than one call component: a trill and a series of chirps. Models of oscillators in chirping

316 species such as Necoconocephalus spiza assume a single oscillator that increases in level and is reset when

317 an external chirp is heard (Greenfield and Roizen, 1993a). In 'Two Part Caller', each calling component

318 could, however, differentially affect the production of calls. In separate playback experiments, we played

319 back both trills and chirps to calling males and examined how they affected calling. The results of our

320 experiments indicate that while trill playback affects the timing and synchrony of trills, chirp playback does

321 not seem to affect them. This suggests that trill production is governed by an oscillator that is unaffected

322 by the chirps. Playback of trills does affect the adjustment of chirp periods, as does playback of chirps. We

323 therefore propose a dual-oscillator model in which the production of trills and chirps are governed by two

324 different oscillators (Fig. 9). During solo calling, the trill oscillator suppresses the production of chirps for

325 the duration of the trill, after which the chirp oscillator produces the chirps. While interacting with another

326 calling male, the trills heard reset the trill oscillator as seen in the trill PRC. The chirps, however, do not

327 reset the trill oscillator but affect the production of chirps by the chirp oscillator if heard in between trills,

328 thus enabling the level of chirp synchrony seen. Such a model would also predict that when a male's trills

329 are suppressed by external trills, the suppression of the focal male's chirps should be released, and we

330 would expect more chirps to be produced in this case. Our results seem to bear this out, since more chirps

331 are produced when external trills extend beyond a male's trills, and thus presumably suppress further trill

332 production. 


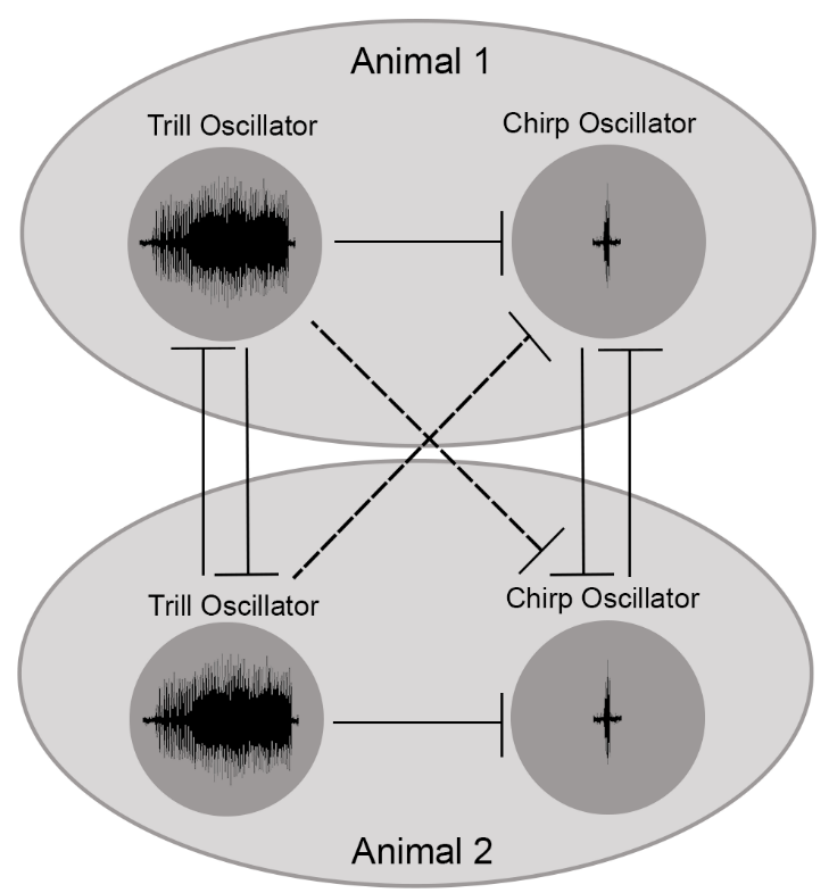

Fig. 9. Two oscillator model for synchrony in Mecopoda 'Two Part Caller'. Circles represent oscillators governing the production of trills and chirps. Larger ellipses represent each of two animals acoustically interacting with each other. Lines depict inhibitory processes. Inhibition between the trill oscillator and chirp oscillator of the other male is indicated as a dotted line to suggest a possibly different process. evolutionarily stable strategy (ESS) in response to such a female preference (Greenfield and Roizen, 1993a; 
are preferentially represented in the auditory system of the female (Römer et al., 2002). The representation effect on the auditory system of the female. If contralateral inhibition mechanisms similar to Mecopoda elongata operate in this species, a male with leading trills could therefore gain a huge advantage - the call of the following male would be inhibited for a long duration during which only the male with the leading trill would be heard. This could mean that females strongly prefer males with leading trills. Such a preference would create a strong selective pressure on the males to produce the same and could have driven the evolution of synchrony in this species in a process like that seen in Necoconocephalus spiza. An alternative evolutionary scenario could be that females prefer calls with chirps, and that the males need to synchronize the trills so that the chirps can be heard in the gaps. There might then be resemblances to the prefer calls with chucks compared to calls without (Ryan, 1980).

To the best of our knowledge, this is the first report of acoustic synchrony of non-isochronous calls in an insect. This suggests that the ability to rhythmically respond to complex stimuli could be more widespread amongst animals than has previously been thought. However, it is important to clarify that the mechanisms underlying such abilities could be very different in different species. Our two-oscillator model suggests that the mechanisms of synchrony effectively reduce the differing rhythms in the call to two separate patterns

367 of trills and chirps and the oscillators respond separately to each of these. This converts what might appear Mecopoda, it seems as if differing neural mechanisms could govern call synchrony. How these mechanisms

371 have evolved and the selective pressures on them makes for a fascinating future area of research. Further 
372

373

374

375

376

377

378

379 studies, for example, are needed to shed light on female preferences for leading trills and the neural representation of these in the nervous system of 'Two Part Caller'. It would also be important to investigate if, despite the similarities in synchronizing behaviour, the processes leading to the evolution of the behaviour in this species could differ, as has been suggested for the species Mecopoda 'Chirper' (Nityananda and Balakrishnan, 2009).

Acknowledgements: VN is currently funded by a Biotechnology and Biological Sciences Research Council David Phillips Fellowship BB/S009760/1. We are grateful to the Ministry of Environment, Forests and Climate Change Government of India for funding this project. The equipment used in the study was funded by a DST-SERB grant to RB. We thank Dr Natasha Mhatre for help with writing the stimulus programs in Labview and Dr Hari Sridhar for help with fieldwork collecting the katydids. The experiments comply with the legal principles ofanimal care and animal welfare of the Government of India.

Data Availability: All data supporting this paper can be accessed using the following link: https://doi.org/10.6084/m9.figshare.13293089.v1.

\section{References}

Alexander, R. D. (1967). Acoustical Communication in Arthropods. Annu. Rev. Entomol. 12, 495-526.

Batschelet, E. (1981). Circular statistics in biology. New York: Academic Press.

Buck, J., Buck, E., Case, J. F. and Hanson, F. E. (1981a). Control of Flashing in Fireflies V. Pacemaker Synchronization in Pteroptyx cribellata. J. Comp. Physiol. A 144, 287-298.

Buck, J., Buck, E., Hanson, F. E., Case, J. F., Mets, L. and Atta, G. J. (1981b). Control of flashing in fireflies IV. Free Run Pacemaking in a Synchronic Pteroptyx. J. Comp. Physiol. A 144, 277-286.

Cabin, R. J. and Mitchell, R. J. (2010). To Bonferroni or Not to Bonferroni : When and How Are the 
Questions. Bull. Ecol. Soc. Am. 81, 246-248.

Celma-Miralles, A. and Toro, J. M. (2018). Beat perception in a non-vocal learner: Rats can identify isochronous beats. $76-79$.

Cook, P., Rouse, A., Wilson, M. and Reichmuth, C. (2013). A California sea lion (Zalophus californianus) can keep the beat: Motor entrainment to rhythmic auditory stimuli in a non vocal mimic. J. Comp. Psychol. 127, 412-427.

Ermentrout, B. (1991). An adaptive model for synchrony in the firefly Pteroptyx malaccae. J. Math. Biol. 29, 571-585.

Fertschai, I., Stradner, J. and Römer, H. (2007). Neuroethology of female preference in the synchronously singing bushcricket Mecopoda elongata (Tettigoniidae; Orthoptera): why do followers call at all? J. Exp. Biol. 210, 465-76.

Greenfield, M. D. (1994). Synchronous and Alternating Choruses in Insects and Anurans: Common Mechanisms and Diverse Functions. Am. Zool. 34, 605-615.

Greenfield, M. D. (2002). Signalers and Receivers: Mechanisms and Evolution of Arthropod Communication. Oxford: Oxford University Press.

Greenfield, M. D. and Roizen, I. (1993a). Katydid synchronous chorusing is an evolutionary stable outcome of female choice. Nature 364, 618-620.

Greenfield, M. D. and Roizen, I. (1993b). Katydid synchronous chorusing is an evolutionary stable outcome of female choice. Nature 364, 618-620.

Greenfield, M. D. and Schul, J. (2008). Mechanisms and evolution of synchronous chorusing: emergent properties and adaptive functions in Neoconocephalus katydids (Orthiptera: Tettigoniidae). J. Comp. Psychol. 122, 289-97. 
Greenfield, M. D. and Snedden, W. A. (2003). Selective attention and the spatio-temporal structure of Orthopteran choruses. Behaviour 140, 1-26.

Greenfield, M. D., Tourtellot, M. K. and Snedden, W. A. (1997). Precedence effects and the evolution of chorusing. Proc. R. Soc. B Biol. Sci. 264, 1355-1361.

Hanson, F. (1978). Comparative studies of firefly pacemakers. Fed. Proc. 37, 2158-2164.

Hartbauer, M., Kratzer, S., Steiner, K. and Römer, H. (2005). Mechanisms for synchrony and alternation in song interactions of the bushcricket Mecopoda elongata (Tettigoniidae: Orthoptera). $J$. Comp. Physiol. A 191, 175-88.

Hasegawa, A., Okanoya, K., Hasegawa, T. and Seki, Y. (2011). Rhythmic synchronization tapping to an audio-visual metronome in budgerigars. Sci. Rep. 1, 120.

Jao Keehn, R. J., Iversen, J. R., Schulz, I. and Patel, A. D. (2019). Spontaneity and diversity of movement to music are not uniquely human. Curr. Biol. 29, R621-R622.

Nityananda, V. and Balakrishnan, R. (2006). A diversity of songs among morphologically indistinguishable katydids of the genus Mecopoda (Orthoptera: Tettigoniidae) from Southern India. Bioacoustics 15, 223-250.

Nityananda, V. and Balakrishnan, R. (2007). Synchrony during acoustic interactions in the bushcricket Mecopoda "Chirper" (Tettigoniidae:Orthoptera) is generated by a combination of chirp-by-chirp resetting and change in intrinsic chirp rate. J. Comp. Physiol. A 193, 51-65.

Nityananda, V. and Balakrishnan, R. (2009). Modeling the role of competition and cooperation in the evolution of katydid acoustic synchrony. Behav. Ecol. 20, 484-489.

Patel, A. D., Iversen, J. R., Bregman, M. R. and Schulz, I. (2009). Experimental Evidence for Synchronization to a Musical Beat in a Nonhuman Animal. Curr. Biol. 19, 827-830. 
Römer, H. and Krusch, M. (2000). A gain-control mechanism for processing of chorus sounds in the afferent auditory pathway of the bushcricket Tettigonia viridissima (Orthoptera; Tettigoniidae). $J$. Comp. Physiol. A 186, 181-91.

Römer, H., Hedwig, B. and Ott, S. R. (2002). Contralateral inhibition as a sensory bias: the neural basis for a female preference in a synchronously calling bushcricket, Mecopoda elongata. Eur. $J$. Neurosci. 15, 1655-1662.

Ryan, M. J. (1980). Female mate choice in a neotropical frog. Science (80-. ). 209, 523-525.

Schachner, A., Brady, T. F., Pepperberg, I. M. and Hauser, M. D. (2009). Spontaneous Motor Entrainment to Music in Multiple Vocal Mimicking Species. Curr. Biol. 19, 831-836.

Sismondo, E. (1990). Synchronous, alternating, and phase-locked stridulation by a tropical katydid. Science (80-. ). 249, 55-8.

Snedden, W. A. and Greenfield, M. D. (1998). Females prefer leading males: relative call timing and sexual selection in katydid choruses. Anim. Behav. 56, 1091-1098.

Wilson, M. and Cook, P. F. (2016). Rhythmic entrainment: Why humans want to, fireflies can't help it, pet birds try, and sea lions have to be bribed. Psychon. Bull. Rev. 23, 1647-1659. 